\title{
The influence of regression curve parameters of creep behaviour on measured data prediction
}

\author{
Martin Reznicek ${ }^{1, a}$, Janostik Vaclav ${ }^{1}$ and Ondrej Bilek ${ }^{1}$ \\ ${ }^{1}$ Tomas Bata University in Zlin, Nam. T.G. Masaryka 5555, Zlín, 76001 Czech Republic
}

\begin{abstract}
This study deals with the study of the influence of regression curve parameters on prediction of possibilities of measured data. In this study, the necessary introduction to polymer materials issues and their material structure which influences their physical and mechanical properties, is described. Thereafter, the method of testing materials using device DMA which enables this measurement is presented. In the next part, this measured data are processed using creep modulus in which regression curves are applied. In the final part of this study, the influence of individual parameters of the regression curve on their process during the prediction is described. The study will prove the inconsiderable influence especially the last parameter of the regression curve equation and its significant influence on measured data prediction.
\end{abstract}

\section{Introduction}

Nowadays, polymeric materials belong to commonly used materials in a number of applications and use. In the practice it is possible to meet a number of methods which enable to study the properties of these materials and to recognize better their behaviour during different conditions of use. These measurements are often unnecessary without the need to choose a certain range of variables which can then be analyzed. It is not possible to always fully influence the choice of the density of this scale, namely the density of the points which are then passed through different lines, curves and splines. However, the density of these points significantly affects the accuracy of the interleaved geometric elements. In interleaving, each used software allows to influence the extent of this interference, and thus the extent to which the interleaved geometry matches the obtained points from the individual measurements. [1-5]

For this purpose, HDPE and LDPE materials from DOW were selected. Polymers are chemicals composed mostly of carbon, hydrogen, oxygen, nitrogen, chlorine and other elements. They are substances which are processed in the liquid state, mostly under elevated temperature and pressure. In this state, it is possible to produce a variety of shapes in the production process. Polymers can be of natural or synthetic origin, from a chemical point of view, they are mainly organic substances composed of large macromolecules. These macromolecules are composed of many repetitive cells in the string called "mer", which is a basic monomeric unit that has the ability to connect under appropriate conditions. From the suffix mer $($ mer $=$ part $)$ and poly

\footnotetext{
a Corresponding author: mreznicek@utb.cz
}

prefix (meaning more from Greek) the word polymer was created.

The length of the macromolecules can be expressed using the molar mass, resp. relative molecular weight. By definition, the macromolecular substance is a compound whose molecular weight exceeds $10^{3} \mathrm{~g} / \mathrm{mol}$, and the polymer bears a substance having a molecular weight greater than $10^{4} \mathrm{~g} / \mathrm{mol}$.

The manufacture of polymers is linked to them. Nowadays, there is an increasing demand for the resulting plastic product. It should have the lowest weight, high temperature resistance, abrasion resistance, frost resistance, it should be firm and tough. These requirements give a permanent impetus to the emergence of new types of polymers, especially high-tech polymers which are now used for example in the aerospace or aerospace industry.[6-9]

Because of different mechanical properties that depend on the internal structure, it is possible to divide the polymers into:

Linear - single monomer molecules join together in a row to form a string. Such polymers have a higher density (e.g. HDPE) due to a spatial arrangement that allows molecules to come closer together.

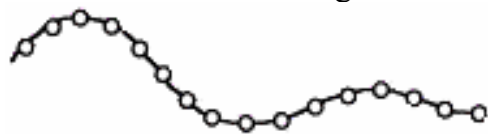

Figure 1. Linear structure

Branched - will occur if side branches are added to the basic string. These branched macromolecules cannot be as close to each other as they are in the linear branches, and thus have a lower density (e.g. LDPE). Due to branching, the mobility of the macromolecules is 
impaired (the molten polymer has lower fluidity) and there is also a decrease in the intermolecular forces (the side chains are further apart) which lead to the worse mechanical properties of the polymer. [10-15]

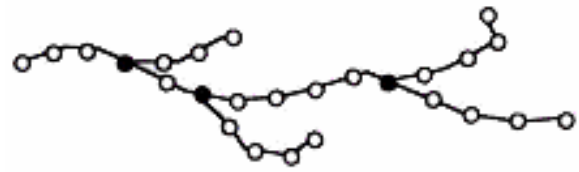

Figure 2. Branched structure

Crosslinked (block, grafted) - are formed by linking straight or branched chains by means of transverse chemical bonds. Such a cross-linked macromolecule has a limited mobility as a whole. This newly formed network results in a change in polymer properties, increases its hardness, stiffness, increased temperature resistance, but resistance to impact stresses is reduced by crosslinking. The resulting polymer is non-melting and insoluble again.

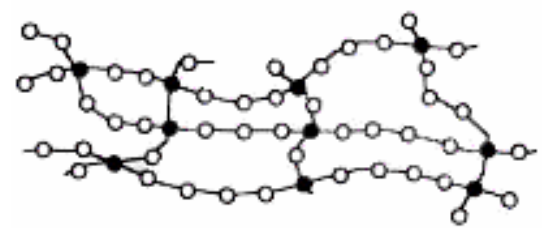

Figure 3. Crosslinked structure

Based on usable properties and possibilities of use, depending on the mechanical properties, the polymeric materials can be assembled into the following pyramid.

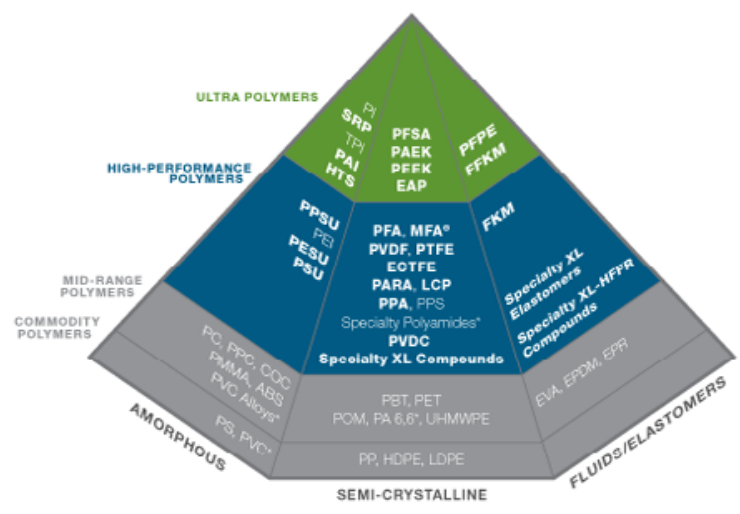

Figure 4. Separation of polymer

\section{Methods}

For creep tests it is necessary to prepare test specimens. These are designed to provide repeatability in predefined shapes and dimensions. For measuring mechanical tests, standard double-sided blades specimens as standard are used, for the measurement of creep properties on the DMA device samples in the shape of a block are used, which can be seen in Fig. 5. This shape and size of samples is adapted for measurement on DMA devices.

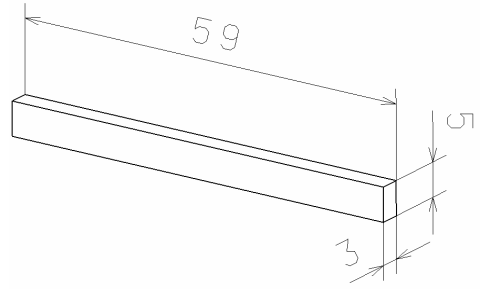

Figure 5. Sample dimensions

The crosslinked material was chosen for the measurement of creep properties for comparison of various options. High-density polyethylene from the company DOW was chosen as a material. Testing samples were made from this material according to a standard ISO 527-2 1BA for the tensile test. The processes of injection moulding and radiation crosslinking were performed with the minimum time gap to avoid the influence on the measurement surroundings.

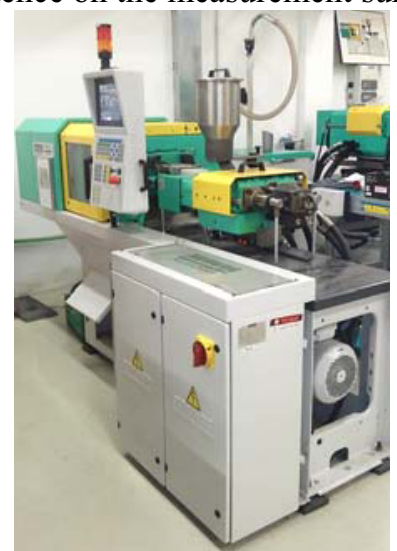

Figure 6. Arburg Allrouder $170 \mathrm{U}$

These samples were moulded by the injection moulding machine ARBURG Allrouder 170U according to the same process conditions.

Table 1. Process conditions

\begin{tabular}{|c|c|}
\hline Parameter & Value \\
\hline $\begin{array}{c}\text { Injection } \\
\text { temperature }\end{array}$ & $200^{\circ} \mathrm{C}$ \\
\hline Barrel temperatures & $195^{\circ} \mathrm{C}, 190^{\circ} \mathrm{C}, 180^{\circ} \mathrm{C}$ \\
\hline Mold temperature & $40^{\circ} \mathrm{C}$ \\
\hline Injection velocity & $40 \mathrm{~mm} / \mathrm{s}$ \\
\hline Injection pressure & $60 \mathrm{MPa}$ \\
\hline Cooling time & $45 \mathrm{~s}$ \\
\hline
\end{tabular}

The samples were irradiated by doses $33 \mathrm{kGy}, 66 \mathrm{kGy}$, 99 kGy, 132 kGy, 165 kGy, 198 kGy. The concurrent measurement followed after irradiation on the device DMA1 from the Company Mettler Toledo and on the device for measurement of creep properties of own produce.

Dynamic mechanical analysis (DMA) is one of the possible methods for measurement of dynamic properties. 
The samples for this kind of test can be exposed to tension, compression, bending and shear.

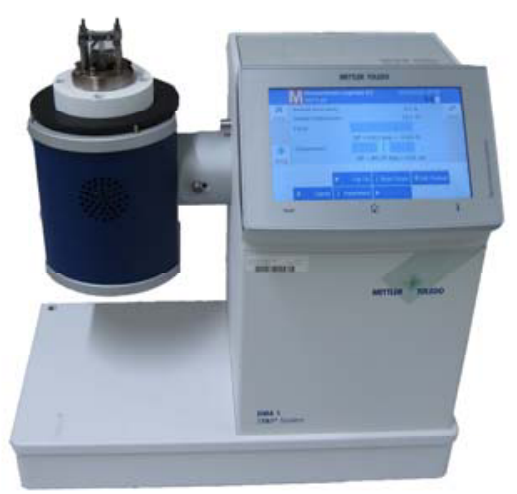

Figure 7. Mettler Toledo DMA1

The device disposes with the mode TMA, which enables to measure the expansion, creep and relaxation time. The device DMA1 in Fig. 7 was used for measurement.

The device DMA1 enables the measurement of brittle and though materials, which are suitable for these type of loading, and it enables evaluation in the program STAR. The load module enables loading from 0.001 to $10 \mathrm{~N}$. The frequency generator enables the variable adjustment of oscillation from 0.001 to $300 \mathrm{~Hz}$.

\section{Data of results}

During evaluating the results, it was necessary to proceed with a slight cut of the measured ones. As can be seen in formula 1 , the resulting module is directly proportional to the loading force and initial length $\mathrm{L}_{0}$ that are constant during measurement and calculation.

$$
E_{t}=\frac{F L_{0}}{A(\Delta L)_{t}}
$$

However, this creep modulus is inversely proportional to the initial cross section of the test piece and, above all, to the elongation value.

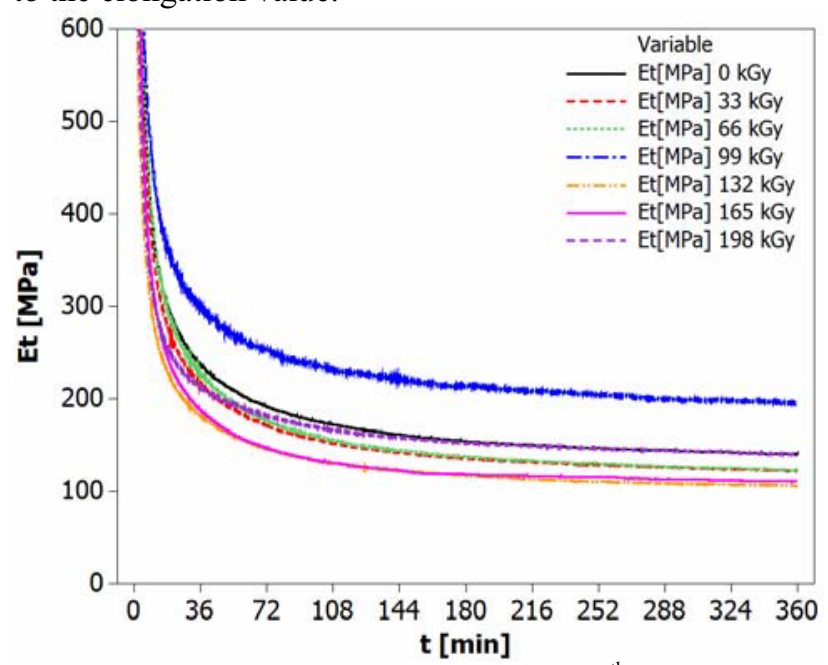

Figure 8.1 HDPE creep tensile modulus from $5^{\text {th }}$.minutes of the measurement
The value of the $\Delta \mathrm{L}$ extension is very small at the beginning of the measurement and the subsequent recalculation of the creep modulus value is thus considerably large up to theoretically infinite. Such high values have a negative effect on the size of the scale, which then does not have sufficient telling value.

The highest initial modulus of elasticity was therefore set to $500 \mathrm{MPa}$, which corresponds approximately to the fifth minute of measurement. This reduction of only $1.3 \%$ value has contributed significantly to better interpretation of the measured data. In Fig. 8, it is possible to see the results of each creep tests. These results have already been confirmed in a number of others and are only a partial confirmation of our correct measurements. As in previous measurements, it is possible to see the dose of $99 \mathrm{kGy}$ as the most appropriate dose of radiation to improve creep properties. Other doses used in these measurements have either minimal or even negative effects on these properties.

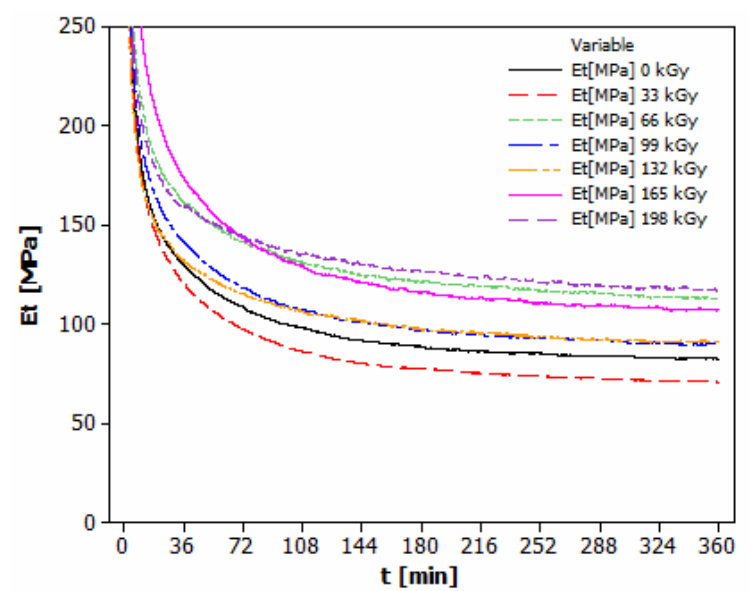

Figure 9. LDPE creep tensile modulus from $5^{\text {th }}$.minutes of the measurement

In Fig. 9, data evaluation for LDPE material irradiated by different doses can be seen. Also in this graph, the initial values were cut at 5 minutes, which significantly contributed to a better interpretation of the measured values. Compared to the results in Fig. 8 a far more compact effect of doses and far less influence on their creep properties can be seen.

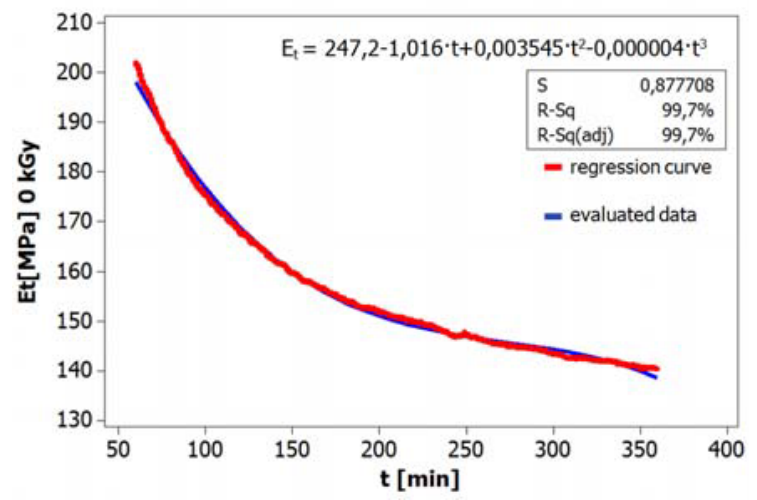

Figure 10. Placing a regression curve with measured HDPE data $0 \mathrm{kGy}$

From the above displayed graph it is possible to see the size of regression curve equations parameters which 
affect the size of the creep tensile modulus. It may seem that parameters (c) and (d) are almost negligible, but with increasing prediction time, these parameters become the determining value of the time.

Table 2. The regression curve reliability

\begin{tabular}{|c|c|c|}
\hline $\begin{array}{c}\text { Radiation dose } \\
\text { [kGy] }\end{array}$ & $\begin{array}{c}\text { Reliability } \\
\text { value (R-Sq) } \\
\text { [\%] HDPE }\end{array}$ & $\begin{array}{c}\text { Reliability } \\
\text { value (R-Sq) } \\
\text { [\%] LDPE }\end{array}$ \\
\hline 0 & 99,7 & 99,6 \\
\hline 33 & 99,6 & 99,4 \\
\hline 66 & 99,5 & 99,6 \\
\hline 99 & 99,4 & 99,6 \\
\hline 132 & 99,5 & 99,5 \\
\hline 165 & 99,2 & 99,5 \\
\hline 198 & 99,7 & 99,5 \\
\hline
\end{tabular}

Table 2 shows the reliability of interleaved regression curves by measured data for individual materials and doses. This reliability was in all cases above the $99 \%$ confidence limit, indicating a high degree of credibility. Fourth degree polynomial was used to find a suitable regression curve.

\section{Conclusion}

The effect of individual regression curve parameters over a short period of time does not greatly influence its behaviour and it is very often adjusted to match the regression curve and the measured data. This method is useful if it is only needed to break the curve with measured data.

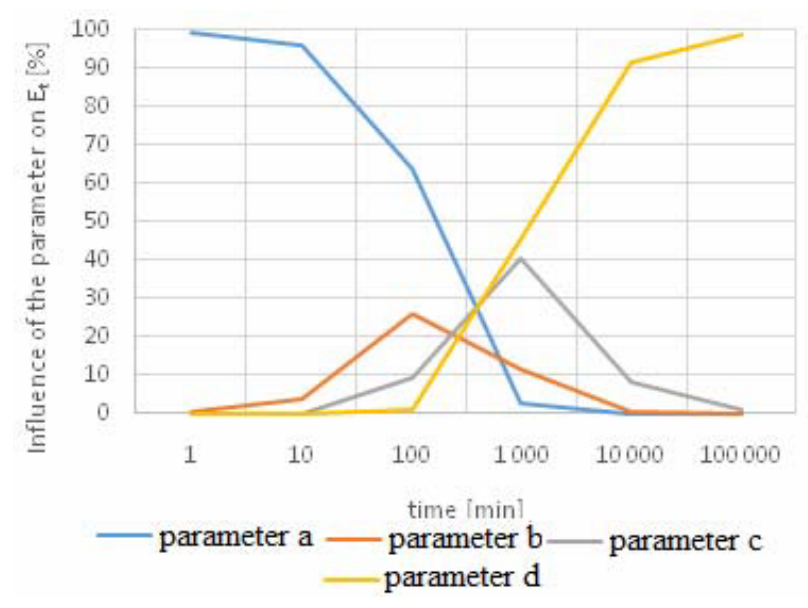

Figure 11. The influence of curve parameters on the prediction

Very often there is an attempt to predict measured data most often in terms of time. Therefore, the measured data is best passed through the regression curve with the parameters completed so that the reliability is as large as possible, and then by simply arriving at the change (e.g. time), it is judged by its behaviour over a longer period of time. However, as seen in Fig. 11, the effect of parameters is variable from the point of view of increasing time, and the initial negligible influence of parameter $\mathrm{d}$ has, with increasing time, a negligible effect on the duration of the regression curve. On the other hand, the initial significant effect of the parameters is reduced to a minimum over time. Parameters $b$ and $c$ have a significant share, especially over the mediumterm. Therefore, when making regression curves, it is necessary to neglect the last parameters of the curve equations describing the data. As proved in this article, its influence is notable in the field of prediction of the measured data behaviour and can significantly affect the conclusions of the individual measurements.

\section{Acknowledgments}

This paper is supported by the internal grant of TBU in Zlin No. IGA/FT/2017/010 funded from the resources of specific university research and by the Ministry of Education, Youth and Sports of the Czech Republic within the National Sustainability Programme project No. LO1303 (MSMT-7778/2014) and also by the European Regional Development Fund under the project CEBIATech No. CZ.1.05/2.1.00/03.0089.

\section{References}

1. Sefidmazgi NR, Bahia HU. Mechanisms of failure in uniaxial repeated creep test and the relationship to aggregate packing. RILEM Bookseries ,11:757-71, (2016).

2. Ren, W., Zhang, D., Wang, G. and CHeng, H. Mechanical and thermal properties of bamboo pulp fiber reinforced polyethylene composites. BioResources, 11, pp. 4117-4127, (2014).

3. Ge H, Le J-, Mantell SC. Numerical modeling of stress corrosion cracking of polymers. Eng Fract Mech, 160:199-212, (2016).

4. E. Morales, J.R. White, J. Mater. Sci., 44 (17) (2009), pp. 4734-4742

5. Ge, H., Li, H., Mantell, S.C.,Annual technical conference - ANTEC, conference proceedings, 2, 1281 - 1286p., (2014).

6. Apollonio C, Covas DIC, de Marinis G, Leopardi A, Ramos HM. Creep functions for transients in HDPE pipes. Urban Water J, 11,:160-6,( 2014).

7. M. Ovsik, D. Manas, M. Manas, M. Stanek, M. Hribova, K. Kocman, D. Samek, Chem. listy, 106 (2012)

8. A. Lalande, D. Gardette Nucl. Instrum. Methods Phys. Res. B, 222 (2004)

9. D. Manas, M. Manas, M. Stanek, M. Danek, Arch. Mater. Sci. Eng., 32 (2) (2008)

10. J.G. Drobny, CRC Press, (2003).

11. O. N. Tretinikov, S. Ogata, Y. Ikada, Polymer 39,24 (1998).

12. G. Zamfirova, V. Gaydarov, T. Zaharescu, L. G. Silva, Chemicke Listy 104 (2010).

13. W.C. Oliver, G.M. Pharr, J. Mater. Res., 19. (2004).

14. D. Manas, M. Hribova, M. Manas, M. Ovsik, Stanek, Thin Solid Films 530 (2013).

15. G. M. Pharr, Materials Science and Engineering (1998). 